\title{
miR-27b is upregulated in cervical carcinogenesis and promotes cell growth and invasion by regulating CDH11 and epithelial-mesenchymal transition
}

\author{
JIHANG YAO, BOYA DENG, LE ZHENG, LEI DOU, YI GUO and KEJUN GUO \\ Department of Gynecology, The First Hospital of China Medical University, Shenyang, Liaoning 110001, P.R. China
}

Received October 2, 2015; Accepted November 5, 2015

DOI: $10.3892 /$ or.2015.4500

\begin{abstract}
Dysregulation of microRNAs (miRNAs) occurs frequently in cervical carcinogenesis. miRNAs function as tumor-suppressors or oncogenes and are involved in tumor behavior. However, the expression and function of miR-27b in cervical carcinogenesis remain unknown. In the present study, we observed that miR-27b was significantly increased in cervical cancer cells and tissues, and upregulation of miR-27b was negatively associated with its direct target, cadherin 11 (CDH11). Upregulation of miR-27b significantly accelerated the proliferation, cell cycle transition from $\mathrm{G} 1$ to $\mathrm{S}$ phase, migration and invasion of $\mathrm{C} 33 \mathrm{~A}$ cells, while downregulation of miR-27b suppressed the proliferation and invasion of $\mathrm{HeLa}$ cells. Moreover, CDH11 cDNA transfection impaired the oncogenic effect of miR-27b on cancer cells. Knockdown of CDH11 attenuated the suppressive effect of an miR-27b inhibitor on cervical cancer cells. In addition, we found that CDH11 was involved in miR-27b-induced epithelial-mesenchymal transition (EMT) by regulating expression of E-cadherin, vimentin and $\mathrm{N}$-cadherin. Our results for the first time indicate that miR-27b acting as an oncogene may play an important role in the progression of cervical cancer by modulating CDH11 and EMT.
\end{abstract}

\section{Introduction}

Cervical cancer is one of the most common gynecological tumors and is the fourth leading cause of tumor-related deaths in women worldwide (1). Although the screening of cervical cancer has been widely utilized, there are still large numbers of patients diagnosed at an advanced stage, particularly in China, with a 5-year survival rate of less than $40 \%$ (2). Moreover, $\sim 30 \%$ of patients suffer from lymph node recurrence and

Correspondence to: Professor Kejun Guo, Department of Gynecology, The First Hospital of China Medical University, 155 Nanjingbei Street, Heping, Shenyang, Liaoning 110001, P.R. China E-mail: kejun_guo@hotmail.com

Key words: epithelial-mesenchymal transition, miR-27b, cervical cancer, CDH11, proliferation, invasion distant metastasis in the period following treatment (3). The underlying molecular pathogenesis of cervical tumorigenesis is complicated and poorly understood.

MicroRNAs (miRNAs) are a group of small RNAs (18-25 nucleotides), which regulate gene expression through sequence-specific pairing of miRNAs with 3'UTR of target mRNAs $(4,5)$. It has been estimated that miRNAs regulate up to one-third of the total human genes, indicating that miRNAs have critical roles in carcinogenesis (6). Dysregulation of miRNAs appears frequently in human malignancies and has been found to correlate with cancer cell proliferation, apoptosis and invasion (7). Several groups have reported that miR-27b is involved in cancer progression as an oncogene or tumor-suppressor gene (8-10). However, the expression and mechanism by which miR-27b exerts its functions in cervical cancer cells remain unclear.

In the present study, it was revealed that miR-27b was overexpressed in cervical cancer cells and tissue specimens, and that downregulation of miR-27b inhibited cervical cancer cell growth and invasion. CDH11 is a direct and functional target of miR-27b and was found to be involved in the functional effect of miR-27b on cell proliferation and invasion. In addition, CDH11 was involved in miR-27b-induced epithelialmesenchymal transition (EMT) by regulating expression of E-cadherin, vimentin, and N-cadherin. Our findings for the first time suggest that miR-27b may act as an oncogene in cervical carcinogenesis by regulating CDH11 and EMT.

\section{Materials and methods}

Samples and cells. The samples of cancer tissue and adjacent non-cancerous tissue were obtained from 37 patients with cervical squamous cell carcinoma who underwent resection surgery between 2010 and 2011 at the Department of Gynecology, The First Hospital of China Medical University. Informed consent from all participants was obtained before surgery, and the present study was approved by the Institutional Review Boards of our hospital. None of the cases received radiotherapy or chemotherapy before the tissues were collected. The Federation of Gynecology and Obstetrics (FIGO) stage of tumors and non-tumor tissues was evaluated and confirmed by two pathologists independently. The obtained tissue specimens were immediately snap-frozen in liquid nitrogen and stored at $-80^{\circ} \mathrm{C}$ until analysis. 
The human cervical carcinoma cells (HeLa, SiHa, CasKi, and $\mathrm{C} 33 \mathrm{~A}$ ) and human immortalized keratinocytes (HaCaT) were obtained from the Cell Resource Center of the Chinese Academy of Sciences (Shanghai, China) and cultured under standard conditions as recommended by the American Type Culture Collection (ATCC; Manassas, VA, USA). All the cell lines were cultured at $37^{\circ} \mathrm{C}$ in a humidified atmosphere with $5 \% \mathrm{CO}_{2}$.

Detection of miRNA and $m R N A$. Total RNA from the cervical cancer cells and tissue specimens was extracted using the TRIzol reagent (Invitrogen, Carlsbad, CA, USA), and $1 \mu \mathrm{g}$ of each total RNA sample was used to synthesize cDNA using the PrimeScript RT reagent kit Perfect Real-Time (Takara, Japan). Total miRNA was extracted using the mirVana miRNA Isolation kit (Ambion, Austin, TX, USA), and cDNA was subsequently synthesized using the SuperScript II Reverse Transcriptase kit (Invitrogen).

Stem-loop RT-PCR was employed for measuring miR-27b expression in the cell lines and tissues as previously described (11). U6 small nuclear RNA was applied as the endogenous control to determine relative miR-27b expression, which was quantified by the $2^{-\Delta \Delta \mathrm{Ct}}$ method. Real-time quantitative PCR (RT-qPCR) was conducted using a FastStart Universal SYBR Green Master kit (Roche, Germany) and amplified with an ABI 7500 Real-Time PCR system (Applied Biosystems, Foster City, CA, USA). $\beta$-actin was used as an internal control for CDH11. Each sample was assayed in triplicate.

Dual-luciferase assay. For the luciferase reporter assay, C33A cells in a 96-well plate were transfected with $50 \mathrm{nM}$ miR-27b mimics or a mimic negative control (mimic NC). Then, the cells were co-transfected with $0.2 \mathrm{mg} / \mathrm{ml}$ of vector with the mutant or wild-type 3'UTR of CDH11. Forty-eight hours later, luciferase activity was assayed using the Dual-Luciferase reporter assay system (Promega, Madison, WI, USA).

Transfection and plasmid construction. miR-27b mimics and miR-27b inhibitor were synthesized by GenePharma (Shanghai, China). Lipofectamine 2000 (Invitrogen) was used for the transfection of the mimics or inhibitor. Cells were seeded in 6-well plates at a concentration of $1 \times 10^{5}$ and maintained in medium without antibiotics for $24 \mathrm{~h}$ before transfection. The miR-27b mimics or inhibitor were transiently transfected into the cells. The medium was replaced with fresh culture medium after $6 \mathrm{~h}$.

CDH11-specific siRNA (Silencer ${ }^{\mathrm{TM}}$ Predesigned siRNA) was purchased from Ambion (Shanghai, China). The CDH11 cDNA was cloned into pcDNA3.1 to construct the CDH11 expression plasmid.

Cell proliferation and cell cycle analysis. Transfected cells were seeded into 96-well plates (2,000 cells/well) and then MTT $(5 \mathrm{mg} / \mathrm{ml})$ was added to each well for $4 \mathrm{~h}$. The reaction was then terminated by removal of the supernatant followed by the addition of $200 \mu \mathrm{l}$ of DMSO, and absorbance readings at $490 \mathrm{~nm}$ were obtained in triplicate. For the cell cycle analysis, the cells were harvested by trypsinization, washed twice and fixed in $70 \%$ ethanol overnight at $4^{\circ} \mathrm{C}$. Then the cells were subsequently incubated with $20 \mathrm{mg} / \mathrm{ml}$ propidium iodide (Sigma) for $20 \mathrm{~min}$, and cell cycle analysis was performed using FACS flow cytometry (BD Biosciences, Franklin Lakes, NJ, USA).

Wound-healing and invasion assays. HeLa cells (4x10\%/well) and C33A cells $\left(4 \times 10^{5} /\right.$ well) were seeded in 12 -well plates, cultured overnight, and transfected with the miR-27b inhibitor or mimics, respectively. When the culture had reached nearly $80 \%$ confluency, the cell layer was scratched with a sterile plastic tip and then was immediately washed with growth medium twice and cultured again in Dulbecco's modified Eagle's medium (DMEM) at $37^{\circ} \mathrm{C}$ for $48 \mathrm{~h}$. Wound healing was measured at 0 and $48 \mathrm{~h}$. The invasion assay of the HeLa and C33A cells was analyzed in Transwell chambers with membranes coated with Cultrex Basement Membrane Extract (R\&D Systems, Minneapolis, MN, USA). For this purpose, $2 \times 10^{4}$ cells transfected with the miR-27b mimic or inhibitor were suspended in $200 \mu \mathrm{l}$ DMEM without serum and seeded on the upper chamber. Forty-eight hours later, cells on the lower side of the membrane were fixed, stained and counted.

Western blot analysis. Total proteins in the transfected HeLa and C33A cells were extracted and then separated using SDS-PAGE. $\beta$-actin was used as a loading control. Specific antibodies for E-cadherin (1:1,500, DECMA-1, sc-59778), vimentin (1:1,500, RV202, sc-32322), and N-cadherin (1:1,500, D-4, sc-8424) (all from Santa Cruz Biotechnology, Santa Cruz, CA, USA) were employed for the immune detection of the corresponding proteins. After blocking with $5 \%$ free-fat milk in Tris-buffered saline/0.05\% Tween-20 (TBST), the membrane was incubated with a specific primary antibody and then the secondary antibody. Proteins were visualized using ECL reagents (Pierce, Rockford, IL, USA).

Statistical analysis. Data are presented as means \pm SD from at least three independent experiments. The Student's t-test was used to analyze differences in experiments with cell lines. Expression of miR-27b and CDH11 in tissues was compared by using a paired-sample t-test. Correlation between expression levels of miR-27b and CDH11 in the cervical cancer tissues was analyzed using Pearson's correlation coefficient. All statistical tests were two-sided, and $\mathrm{p}<0.05$ was considered to indicate a statistically significant difference.

\section{Results}

miRNA-27 directly targets CDH11 in cervical cancer cells and tissues. miR-27b has been reported as a tumor-suppressor or an oncogene based on different malignancies. However, its role in cervical carcinogenesis remains unclear. Therefore, we initially verified the level of miR-27b in cervical cancer and HaCaT cells. As shown in Fig. 1A, expression of miR-27b was significantly increased in the cervical cancer cells compared with the HaCaT cells. The expression of miR-27b in the tumors was markedly higher in comparison to the adjacent normal tissues $(0.244 \pm 0.084$ vs. $0.199 \pm 0.075, p=0.001$; Fig. 1B).

Three publically available bioinformatic databases, TargetScan, PicTar, and miRanda, were used to predict the potential targets of miR-27b. All three databases predicted 

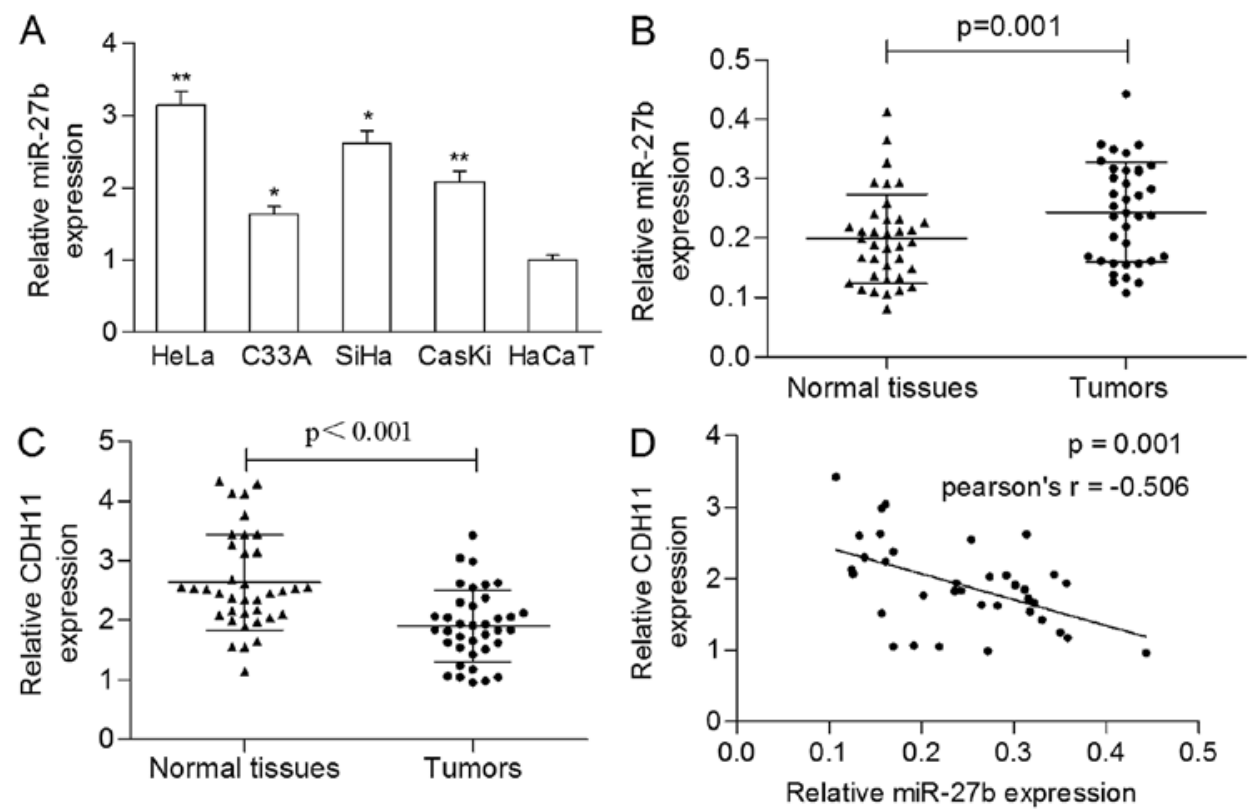

Figure 1. miRNA-27b is upregulated in cervical cancer and is negatively correlated with CDH11. Expression levels of miR-27b in (A) cervical cancer cell lines (HeLa, C33A, SiHa and CasKi) and a normal control cell line (HaCaT) and in (B) 37 paired cervical cancer tissues, respectively. (C) mRNA level of CDH11 in cervical cancer tissues. (D) miR-27b was found to be negatively correlated with the mRNA level of CDH11 in the cancerous tissues.
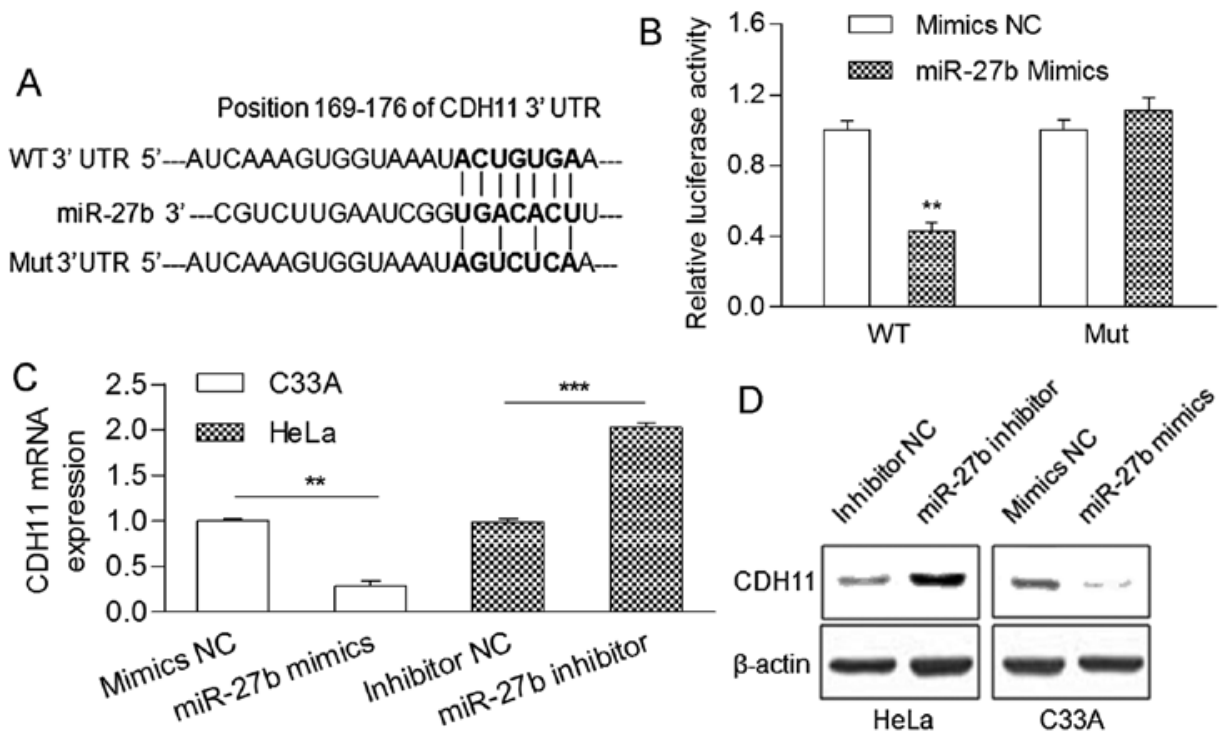

Figure 2. CDH11 is a direct target of miR-27b. (A) miR-27b and its putative binding sites in the 3'UTR of CDH11. Mutation was generated in the CDH11 3'UTR by mutating seed matching sequence. (B) Luciferase report assay was performed to confirm the miR-27b binding target. (C and D) The relative expression levels of CDH11 mRNA and protein in the HeLa and C33A cells after $24 \mathrm{~h}$ post-transfection of the miR-27b inhibitor and mimics, respectively.

CDH11 as a putative target of miR-27b. Thus, we detected the mRNA level of CDH11 in clinical samples and found that the expression of CDH11 was significantly reduced in the primary tumors compared to that in the matched nonmalignant tissues $(1.907 \pm 0.600$ vs. $2.631 \pm 0.800$, p $<0.001$; Fig. 1 C). A significantly negative correlation was found between the expression of miR-27b and the mRNA level of CDH11 in the cancerous tissues (Pearson $r=-0.506, p=0.001$; Fig. 1D).

As shown in Fig. 2A, a highly conserved position (169-176 bp) in the CDH11 3'UTR was predicted to be a binding site of miR-27b. Then, a luciferase reporter assay showed that the relative luciferase activity of the reporter gene with the wild-type CDH11 3'UTR was significantly decreased in the presence of the miR-27b mimic (Fig. 2B). Moreover, upregulation of miR-27b by transfection with mimics in the HeLa cells reduced the mRNA and protein levels of CDH11, while downregulation of miR-27b by transfection with an inhibitor in the C33A cells increased mRNA and protein levels (Fig. 2C and D), indicating that miR-27b directly targets the CDH11 3'UTR.

Effect of miR-27b on cervical cancer cell proliferation and invasion. To analysis the function of miR-27b in cervical cancer cell growth, HeLa cells expressing a relatively high 
A

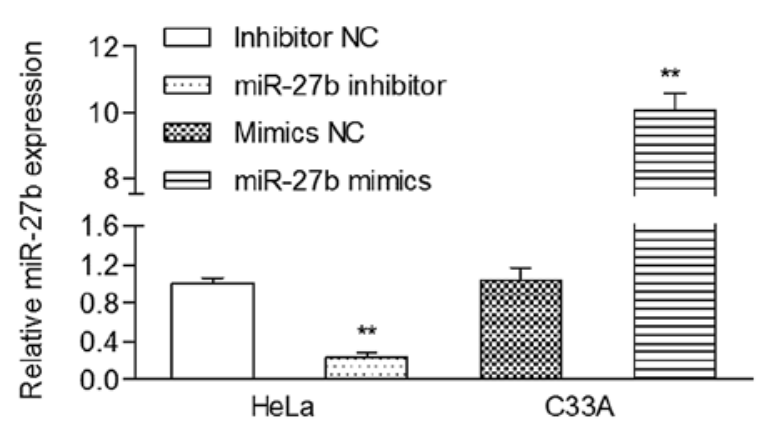

B
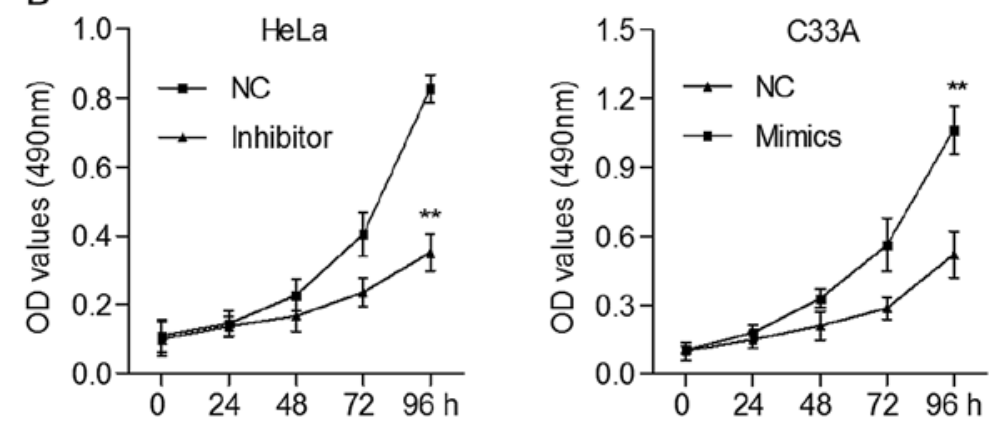

C
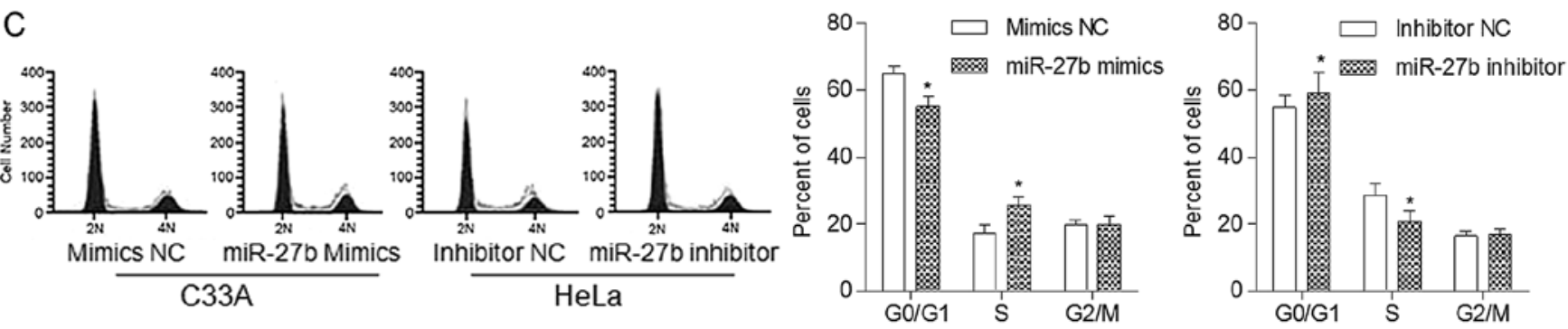

$\mathrm{D}$
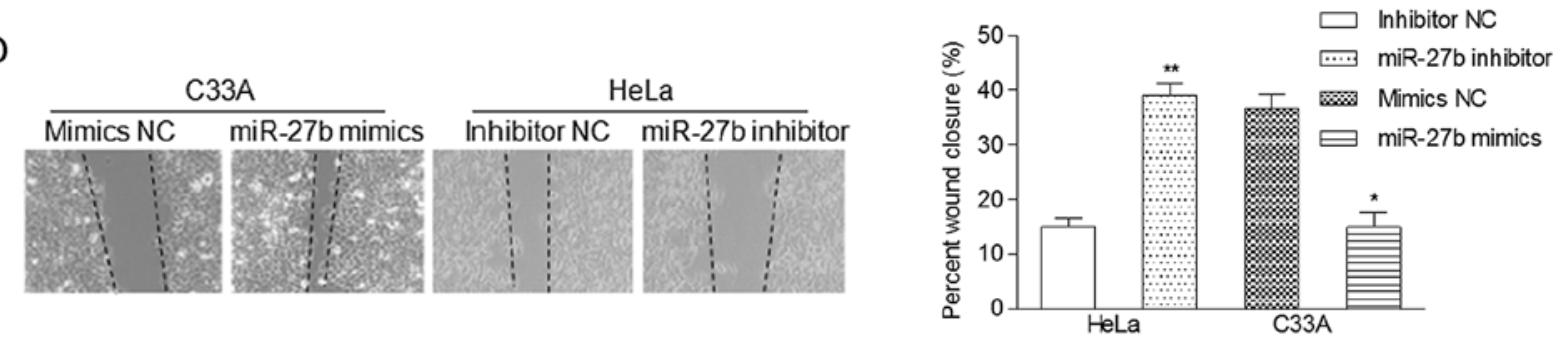

$E$
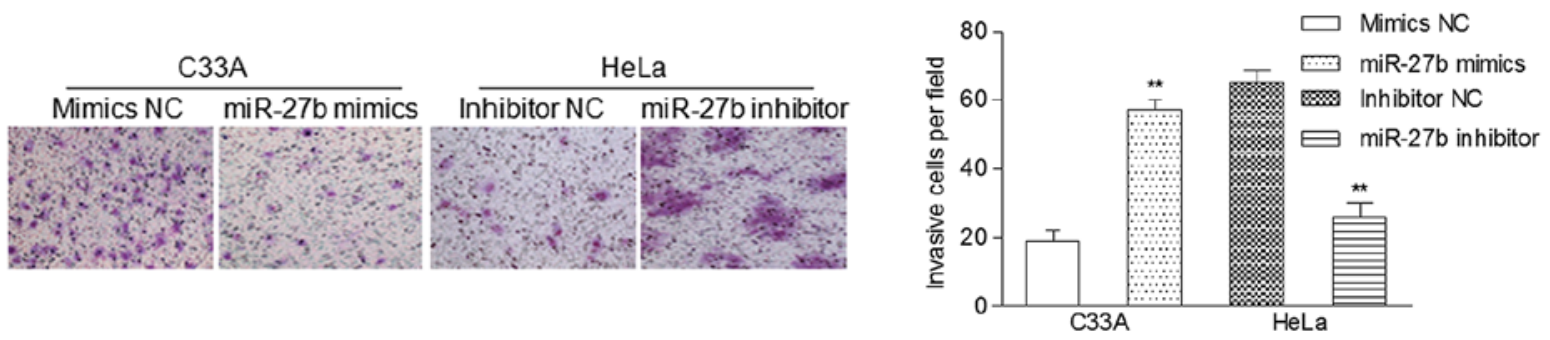

Figure 3. miR-27b promotes cervical cancer cell proliferation and invasion. (A) RT-qPCR analysis of miR-27b levels in the HeLa and C33A cells transfected with the miR-27b inhibitor and miR-27b mimics, respectively. (B) Detection of cell proliferation using the MTT assay. (C) Determination of cell cycle distribution by flow cytometry. (D) Analysis of the migration with the wound-healing assays. (E) Analysis of cell invasion with the Transwell invasion assay. ${ }^{*} \mathrm{p}<0.05,{ }^{* *} \mathrm{p}<0.01$.

level of endogenous miR-27b and C33A cells expressing a relatively low level of endogenous miR-27b were transfected with the miR-27b inhibitor and miR-27b mimics, respectively. The level of miR-27b was significantly decreased in the HeLa cells and increased in the C33A cells when compared to the negative control (Fig. 3A). MTT analysis showed that upregulation of miR-27b significantly promoted the proliferation rate of the C33A cells, while downregulation of miR-27b suppressed the proliferation of the HeLa cells (Fig. 3B). To further understand the mechanism of how miR-27b accelerated cell proliferation, we subsequently studied whether miR-27b plays a role in cell cycle progression. We found that upregulation of miR-27b in the C33A cells led to a significant reduction in the cellular population in the G0/G1 phase but a sharp increase in the cell population in the $\mathrm{S}$ phase, while suppression of miR-27b in HeLa cells obviously induced
G1 phase arrest (Fig. 3C). Therefore, the enhancement of cell cycle progression at the G1/S transition may be responsible for the growth-promotion role of miR-27b in cervical cancer cells.

The migratory capability of the HeLa and C33A cells after transfection was measured using a wound-healing assay. We observed that miR-27b inhibitor-transfected HeLa cells had a notably depressed migratory ability in comparison with the control cells. miR-27b mimic-transfected C33A cells displayed an enhanced migratory capability when compared with the control cells (Fig. 3D). Moreover, the Matrigel Transwell assay showed that miR-27b mimics obviously increased the invasive capability of the C33A cells, whereas the miR-27b inhibitor suppressed this action in the HeLa cells (Fig. 3E). These results strongly indicate that miR-27b exerts an oncogenic effect on cervical carcinogenesis. 

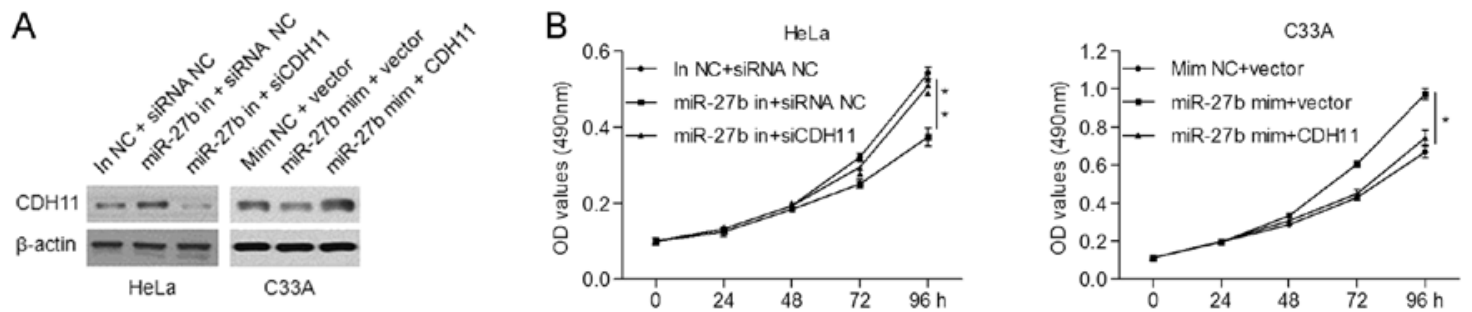

C

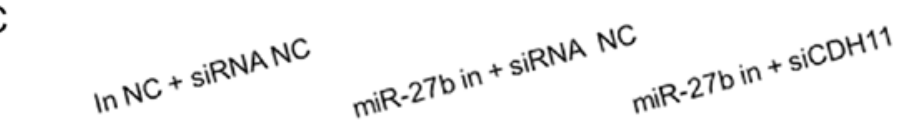
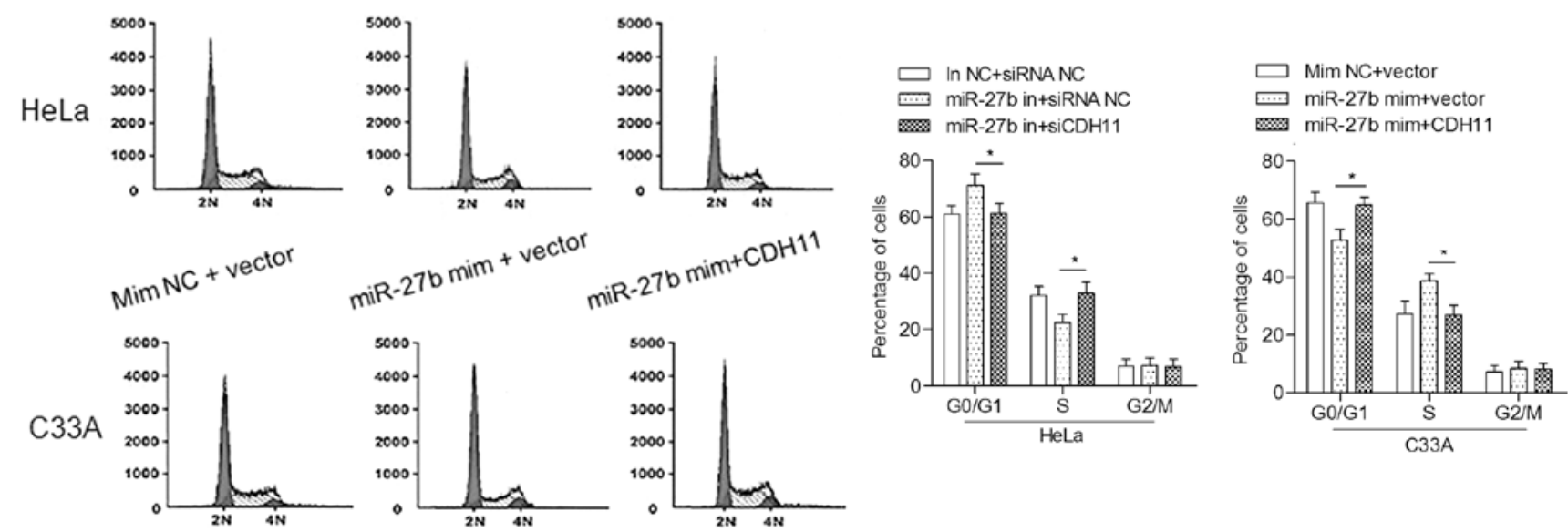

D
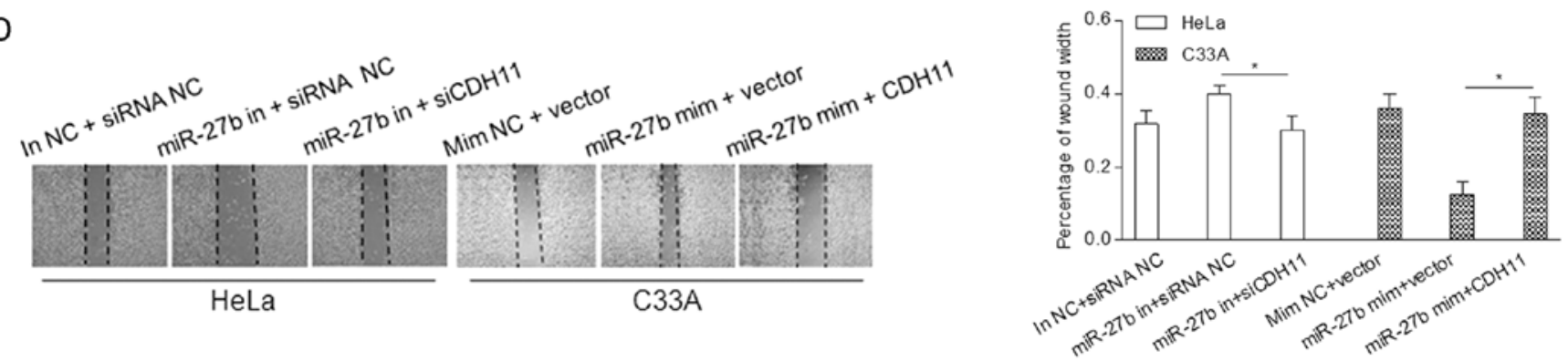

$\mathrm{E}$
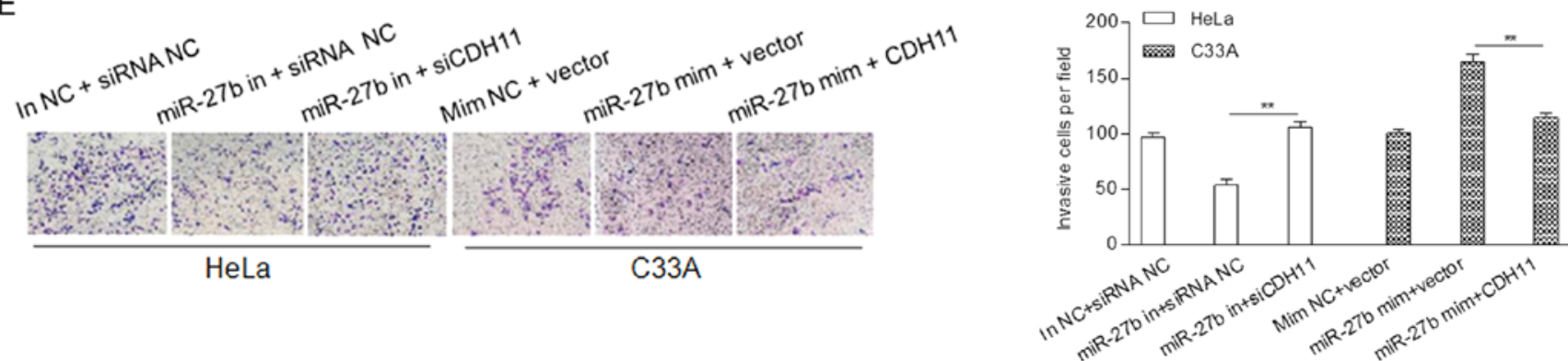

Figure 4. CDH11 is involved in miR-27b-regulated cell proliferation and invasion. HeLa cells were transfected with the miR-27b inhibitor or co-transfected with the miR-27b inhibitor and CDH11-specific siRNAs (siCDH11), while C33A cells were transfected with the miR-27b mimics or co-transfected with the miR-27b mimics and the CDH11 cDNA vector (CDH11). (A) Western blot analysis of CDH11 expression in the HeLa and C33A cells. (B) Analysis of cell proliferation using the MTT assay. (C) Flow cytometric analysis of the cell cycle. (D) Analysis of cell migration using a wound-healing sassy. (E) Analysis of cell invasion ability using the Transwell assay. ${ }^{*} \mathrm{p}<0.05,{ }^{* *} \mathrm{p}<0.01$.

CDH11 is involved in miR-27b-induced effects on cervical cancer proliferation and invasion. To further explore that miR-27b may be involved in the promotion of cell proliferation, migration and invasion by modulating CDH11, we co-transfected the HeLa cells with an miR-27b inhibitor and siCDH11, and the C33A cells with miR-27b mimics and CDH11 cDNA, in which the expression of $\mathrm{CDH} 11$ was determined by western blot analysis (Fig. 4A).

MTT assay showed that knockdown of CDH11 significantly reversed the inhibitory effect of the miR-27b inhibitor on cell proliferation of the HeLa cells. Overexpression of CDH11 markedly inhibited the miR-27b-induced proliferation of the 
A
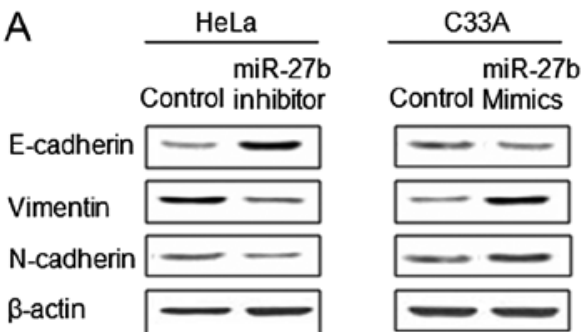

B

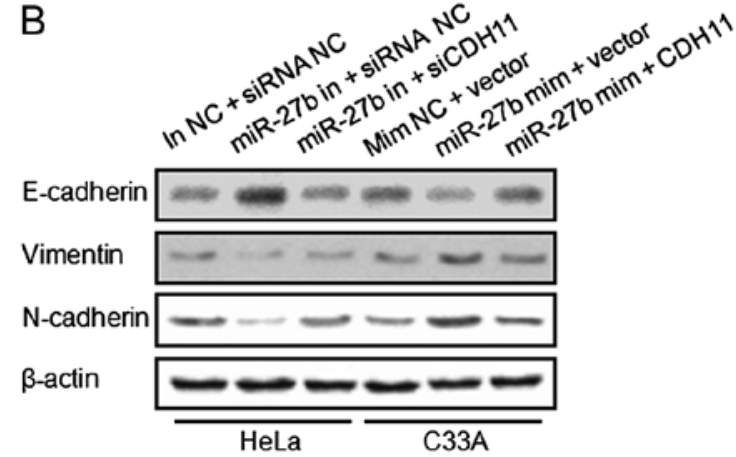

Figure 5. miR-27b induces epithelial-mesenchymal transition (EMT) of cervical cancer cells. (A) HeLa and C33A cells were transfected with the miR-27b inhibitor or miR-27b mimics and the levels of E-cadherin, vimentin and $\mathrm{N}$-cadherin protein were analyzed by western blot analysis. (B) The expression of EMT-related proteins was determined by western blot analysis.

C33A cells (Fig. 4B). Furthermore, upregulation of CDH11 markedly abrogated the enhancement of cell cycle progression at the G1/S transition induced by miR-27b in the C33A cells, whereas knockdown of CDH11 rescued the G1 phase arrest induced by the miR-27b inhibitor in the HeLa cells (Fig. 4C).

In addition, wound-healing and invasion assays indicated that the upregulation of CDH11 attenuated miR-27b-induced migration and invasion of the C33A cells, while silencing of CDH11 impaired the tumor suppressive role of the miR-27b inhibitor on the HeLa cells (Fig. 4D and E). Altogether, these data suggest that $\mathrm{CDH} 11$ is a functional target of miR-27b, involved in miR-27b-induced proliferation and invasion of cervical cancer cells.

$m i R-27 b$ induces $E M T$. For exploring the underlying mechanism of the promotion effect of miR-27b on migration and invasion, we further analyzed classical markers of EMT in HeLa and C33A cells. As shown in Fig. 5A, the miR-27b inhibitor led to elevated expression of E-cadherin and reduced expression of vimentin and $\mathrm{N}$-cadherin in the HeLa cells when compared with levels in the control cells. In the transfected miR-27b mimic C33A cells, we observed a decreased expression of E-cadherin and enhanced expression of vimentin and $\mathrm{N}$-cadherin in the C33A cells when compared with these levels in the control cells. These data suggest that activation of EMT at least partially explains the miR-27b-induced migration and invasion of cervical cancer cells.

Moreover, we detected the expression of EMT-related proteins in the knockdown and overexpressing CDH11 cells, respectively. The expression of E-cadherin was notably reduced, while the expression of vimentin and $\mathrm{N}$-cadherin was increased in the HeLa cells co-transfected with siCDH11 and the miR-27b inhibitor when compared to the cells transfected with the inhibitor alone. E-cadherin was recovered, and vimentin and $\mathrm{N}$-cadherin were suppressed in the $\mathrm{C} 33 \mathrm{~A}$ cells co-transfected with the miR-27b mimic and CDH11 in comparison with levels in the control cells (Fig. 5B). These data suggest that CDH11 is involved in miR-27b-regulated EMT.

\section{Discussion}

Exploration of novel miRNAs involved in carcinogenesis would provide new insights for the diagnostic application and therapeutic advances to benefit cervical cancer patients. Our study demonstrated for the first time to the best of our knowledge that miR-27b plays an oncogenic role in cervical tumorigenesis.

In the present study, we detected the expression level of miR-27b in cervical cancer cells and tissues, and found that miR-27b was significantly upregulated in the tumors, suggesting an oncogenic role of miR-27b in the development of cervical cancer. Notably, miR-27b expression patterns differ among human cancers. miR-27b was found to be upregulated in clinical samples of glioma and hepatocellular carcinoma $(8,12)$, whereas its downregulation occurred in clear cell renal cell carcinoma (10). More importantly, the level of miR-27b was significantly increased in metastatic breast cancer cases and invasive endometrioid endometrial adenocarcinomas $(13,14)$. Thus, we hypothesized that miR-27b may be an invasion-related gene in cancer. The relationship between upregulation of miR-27b and clinicopathological features, such as lymph node metastasis and distant metastasis, require further research in a large cohort of cervical cancer cases.

Functionally, the results of the MTT assay showed that miR-27b significantly promoted the proliferation of C33A cells. miR-27b overexpression led to an accelerated rate of cell migration and invasion in the C33A cells. We observed a tumor-suppressive role of the miR-27b inhibitor on the proliferation and migration of HeLa cells, suggesting that downregulation of miR-27b may be used as a novel therapeutic approach for the treatment of cervical cancer. In accordance with our data, growth suppression and inhibition of invasion were induced by loss of function of miR-27b in glioma cells (8). Wang et al reported that anti-miR-27b inhibited cell invasion in MDA-MB-231 cells, while pre-miR-27b promoted invasion (9). However, it cannot be ignored that miR-27b has been reported as a tumor-suppressor due to its inhibitory effect on cell proliferation and migration $(10,15,16)$. These controversial studies suggest that miR-27b functions as a tumor-suppressor or oncogene depending on tumor type and its target in different tumors. Our data provide initial evidence that miR-27b is an oncogene in cervical carcinogenesis by accelerating proliferation and invasion.

EMT phenotypes have been reported to occur in chemoresistant human cancer cells, and the gain of the EMT phenotype is correlated with the migratory ability of cancer cells (17). In the present study, we revealed for the first time that miR-27b induced EMT of cervical cancer cells through downregulation of E-cadherin and upregulation of vimentin and $\mathrm{N}$-cadherin expression. These data suggest that miR-27b may play a part in EMT of cervical carcinogenesis, which is consistent with previous studies $(18,19)$. Moreover, many studies have found that overexpression of miR-27b is correlated 
with drug-resistance in different types of cancer $(12,20,21)$. Further analysis regarding the relationship between miR-27b and chemoresistance may provide a valuable approach for the chemotherapy of cervical cancer.

Furthermore, we identified CDH11 as a direct target of miR-27b, showing a negative correlation between the levels of miR-27b and CDH11 mRNA in primary tumor tissues. CDH11 a member of the cadherin superfamily, is an integral membrane protein that regulates cell-cell adhesion. The expression of CDH11 is downregulated in osteosarcoma (22) and breast cancer (23). More importantly, several molecular studies have confirmed that CDH11 is a novel invasion-related gene in many types of cancers (23-25). Our data provide evidence that CDH11 is involved in invasion probably through the regulation of EMT, and counteracts the oncogenic effects of miR-27b in cervical cancer cells. Taken together, our data suggest that CDH11 is an invasion-related gene and participates in miR-27b-regulated carcinogenesis.

In conclusion, miR-27b was significantly upregulated in human cervical cancer tissues, and upregulation of miR-27b contributed to cell proliferation and invasion. In addition, CDH11 was identified as a direct target of miR-27b and is involved in miR-27b-induced carcinogenesis and EMT, providing new clues for the treatment of metastatic cervical cancer. Therefore, our results indicate that miR-27 functions as an oncogene partially by modulating CDH11 and can be utilized as a potential therapeutic target in the treatment of cervical cancer.

\section{Acknowledgements}

This study was supported by grants from the National Natural Scientific Foundation of China (no. 81171649).

\section{References}

1. Forouzanfar MH, Foreman KJ, Delossantos AM, Lozano R, Lopez AD, Murray CJ and Naghavi M: Breast and cervical cancer in 187 countries between 1980 and 2010: A systematic analysis. Lancet 378: 1461-1484, 2011.

2. American Cancer Society: Cervical Cancer. American Cancer Society, Atlanta, GA, 2013.

3. Waggoner SE: Cervical cancer. Lancet 361: 2217-2225, 2003.

4. He L and Hannon GJ: MicroRNAs: Small RNAs with a big role in gene regulation. Nat Rev Genet 5: 522-531, 2004

5. Filipowicz W, Bhattacharyya SN and Sonenberg N: Mechanisms of post-transcriptional regulation by microRNAs: Are the answers in sight? Nat Rev Genet 9: 102-114, 2008.

6. Mukherji S, Ebert MS, Zheng GX, Tsang JS, Sharp PA and van Oudenaarden A: MicroRNAs can generate thresholds in target gene expression. Nat Genet 43: 854-859, 2011.

7. Calin GA and Croce CM: MicroRNA signatures in human cancers. Nat Rev Cancer 6: 857-866, 2006.

8. Chen L, Li H, Han L, Zhang K, Wang G, Wang Y, Liu Y, Zheng Y, Jiang T, Pu P, et al: Expression and function of miR-27b in human glioma. Oncol Rep 26: 1617-1621, 2011.

9. Wang Y, Rathinam R, Walch A and Alahari SK: ST14 (suppression of tumorigenicity 14) gene is a target for miR-27b, and the inhibitory effect of ST14 on cell growth is independent of miR-27b regulation. J Biol Chem 284: 23094-23106, 2009.
10. Ishihara T, Seki N, Inoguchi S, Yoshino H, Tatarano S, Yamada Y, Itesako T, Goto Y, Nishikawa R, Nakagawa M, et al: Expression of the tumor suppressive miRNA-23b/27b cluster is a good prognostic marker in clear cell renal cell carcinoma. J Urol 192: 1822-1830, 2014

11. Chen C, Ridzon DA, Broomer AJ, Zhou Z, Lee DH, Nguyen JT, Barbisin M, Xu NL, Mahuvakar VR, Andersen MR, et al: Real-time quantification of microRNAs by stem-loop RT-PCR. Nucleic Acids Res 33: e179, 2005.

12. Zhuo L, Liu J, Wang B, Gao M and Huang A: Differential miRNA expression profiles in hepatocellular carcinoma cells and drug-resistant sublines. Oncol Rep 29: 555-562, 2013.

13. Shen S, Sun Q, Liang Z, Cui X, Ren X, Chen H, Zhang X and Zhou Y: A prognostic model of triple-negative breast cancer based on miR-27b-3p and node status. PLoS One 9: e100664, 2014.

14. Mozos A, Catasús L, D'Angelo E, Serrano E, Espinosa I, Ferrer I, Pons $\mathrm{C}$ and Prat J: The FOXO1-miR27 tandem regulates myometrial invasion in endometrioid endometrial adenocarcinoma. Hum Pathol 45: 942-951, 2014.

15. Jiang J, Lv X, Fan L, Huang G, Zhan Y, Wang M and Lu H: MicroRNA-27b suppresses growth and invasion of NSCLC cells by targeting Sp1. Tumour Biol 35: 10019-10023, 2014.

16. Goto Y, Kojima S, Nishikawa R, Enokida H, Chiyomaru T, Kinoshita T, Nakagawa M, Naya Y, Ichikawa T and Seki N: The microRNA-23b/27b/24-1 cluster is a disease progression marker and tumor suppressor in prostate cancer. Oncotarget 5: 7748-7759, 2014

17. Findlay VJ, Wang C, Watson DK and Camp ER: Epithelial-to-mesenchymal transition and the cancer stem cell phenotype: Insights from cancer biology with therapeutic implications for colorectal cancer. Cancer Gene Ther 21: 181-187, 2014.

18. Cai ZG, Zhang SM, Zhang H, Zhou YY, Wu HB and Xu XP: Aberrant expression of microRNAs involved in epithelial-mesenchymal transition of HT-29 cell line. Cell Biol Int 37: 669-674, 2013.

19. Susuki D, Kimura S, Naganuma S, Tsuchiyama K, Tanaka T, Kitamura N, Fujieda S and Itoh $\mathrm{H}$ : Regulation of microRNA expression by hepatocyte growth factor in human head and neck squamous cell carcinoma. Cancer Sci 102: 2164-2171, 2011.

20. Park YT, Jeong JY, Lee MJ, Kim KI, Kim TH, Kwon YD, Lee C, Kim OJ and An HJ: MicroRNAs overexpressed in ovarian ALDH1-positive cells are associated with chemoresistance. J Ovarian Res 6: 18, 2013

21. Mu W, Hu C, Zhang H, Qu Z, Cen J, Qiu Z, Li C, Ren H, Li Y, He $\mathrm{X}$, et al: $\mathrm{miR}-27 \mathrm{~b}$ synergizes with anticancer drugs via $\mathrm{p} 53$ activation and CYP1B1 suppression. Cell Res 25: 477-495, 2015.

22. Nakajima G, Patino-Garcia A, Bruheim S, Xi Y, San Julian M, Lecanda F, Sierrasesumaga L, Müller C, Fodstad O and Ju J: CDH11 expression is associated with survival in patients with osteosarcoma. Cancer Genomics Proteomics 5: 37-42, 2008.

23. Marino N, Collins JW, Shen C, Caplen NJ, Merchant AS, Gökmen-Polar Y, Goswami CP, Hoshino T, Qian Y, Sledge GW Jr, et al: Identification and validation of genes with expression patterns inverse to multiple metastasis suppressor genes in breast cancer cell lines. Clin Exp Metastasis 31: 771-786, 2014.

24. Delic S, Lottmann N, Jetschke K, Reifenberger G and Riemenschneider MJ: Identification and functional validation of CDH11, PCSK6 and SH3GL3 as novel glioma invasion-associated candidate genes. Neuropathol Appl Neurobiol 38: 201-212, 2012.

25. Li L, Ying J, Li H, Zhang Y, Shu X, Fan Y, Tan J, Cao Y, Tsao SW, Srivastava G, et al: The human cadherin 11 is a pro-apoptotic tumor suppressor modulating cell stemness through Wnt $/ \beta$-catenin signaling and silenced in common carcinomas. Oncogene 31: 3901-3912, 2012. 